\title{
Why do children decide not to participate in clinical research: a quantitative and qualitative study
}

\author{
Irma M. Hein ', Pieter W. Troost ${ }^{1}$, Martine C. de Vries ${ }^{2}$, Catherijne A.J. Knibbe ${ }^{3}$, Johannes B. van Goudoever ${ }^{4,5}$ and \\ Ramón J.L. Lindauer ${ }^{1}$
}

BACKGROUND: More pediatric drug trials are needed, but although specific pediatric regulations warrant safety, recruitment of children for these trials remains one of the main difficulties. Therefore, we investigated potential determining factors of nonparticipation in clinical research, in order to optimize research participation of children by recommending improved recruitment strategies.

METHODS: Between 1 January 2012 and 1 January 2014, we performed a prospective study among161 pediatric patients, aged 6 to $18 \mathrm{y}$, who were eligible for clinical research. We quantitatively analyzed the association of potential explanatory variables (e.g., age, cognitive development, experience, ethnicity) with nonparticipation and qualitatively analyzed interviews on reasons for nonparticipation.

RESULTS: Sixty percent of the children did not participate in the research project on offer (39\% decided not to participate, $21 \%$ were indecisive). Lower age, less disease experience, and less complex research with lower risk were predictive for not participating. Time constraint and extra burden were expressed as decisive reasons for not participating.

CONCLUSIONS: Strategies to optimize research participation should be aimed at younger children and their families, who are logistically challenged and unfamiliar with health care and research. Recommendations include informing pediatric patients and their families of the value of research; minimizing logistic burdens; and improving accessibility.

$\mathbf{M}$ ore drug researches in children are needed, because currently $36-90 \%$ of drugs prescribed to children have not been tested in their age group (1). Prescribers often have no alternative but to resort to off-label or unauthorized products, without having evidence-based information regarding drug effects on children to guide them and help them weigh the risks and benefits of the drug. Conducting more clinical research in children will mean an increased demand for children to participate. Evidently research with this vulnerable population involves many unique ethical and legal considerations which have led to the development of specific pediatric regulations and guidelines to warrant safety.

Research projects need approval at different levels before implementation. Legislative regulations in the United States prescribe that pediatric research that does not offer participants a potential clinical benefit is only allowed when there is (minor increase over) minimal risk and minimal burden (2). Research that may offer the participant potential benefit is assessed by weighing risks and burden against potential gain. Institutional review boards or research ethics committees are charged with the assessment of the research protocols. Informed consent or assent is required from research subjects and their proxies. In the current Code for Federal regulations of the United States (3) by definition children are "persons who have not attained the legal age for consent to procedures involved in the research" (45CRF46.402(a)). Parents are required to provide permission, while regulations state that some children might be able to give their assent, meaning an affirmative agreement. Some authors suggested that children's assent should only be required from a fixed age of $14 \mathrm{y}(4)$, while others argue that children are generally competent to consent from the age of $12 \mathrm{y}$ (5). This illustrates the ongoing debate on child consent issues.

Significant difficulties occur in the recruitment for clinical research involving minors (6). A recent study showed that less than half of the clinical research projects approved by the institutional review board succeeded in procuring $80 \%$ of confirmed participants at the planned closing date. While a consistent pattern of trial characteristics associated with successful recruitment could not be identified (7), previous studies show increasing evidence for the complexity that underpins adult participants' decisions to consent in research. Evidently, adults base their decision on a combination of practical considerations, levels of interest, relational factors, and more general values like a sense of responsibility to contribute knowledge to the community (8).

At present, empirical evidence on what children and parents conceive as burdensome and what they appreciate in research participation is limited. Reasons for, or against, participation

\footnotetext{
'Department of Child and Adolescent Psychiatry, Academic Medical Center, Amsterdam, The Netherlands; ${ }^{2}$ Department of Medical Ethics and Health Law, Leiden University Medical Center, Leiden, The Netherlands; ${ }^{3}$ Department of Clinical Pharmacy, St. Antonius Hospital, Nieuwegein, The Netherlands; ${ }^{4}$ Department of Pediatrics, Emma Children's Hospital, Academic Medical Center, Amsterdam, The Netherlands; ${ }^{5}$ Department of Pediatrics, VU University Medical Center, Amsterdam, The Netherlands. Correspondence: Irma Hein (i.hein@debascule.com) 


\section{Articles $\mid$ Hein et al.}

may be influenced by ethnic status, societal factors, and anxiety or stress, and concrete barriers such as time commitment, childcare, and transportation difficulties (9). Providing participants with inadequate information concerning the particulars of the research also interferes with the participants' ability to make well-considered decisions (10). Studies in adolescents, one of which concerned a hypothetical research project, demonstrated that several participants expected direct health benefit from being questioned and examined by the physician (11-13). A main reason for participation was charity (14), and half of the eligible subjects refused for no apparent reason (15). Sample sizes of the studies are small, children under $12 \mathrm{y}$ are underrepresented, and studies do not show results for subdivisions of children in different age groups. Furthermore, answers of children might be biased by social desirability. Combining qualitative and quantitative outcomes can offer a more comprehensive insight into factors that promote participation as well as factors that militate against it.

Insight into motives and characteristics of children who decide not to participate in research is of importance for the development of strategies to improve recruitment rates and by that increase successful conduct of research. The aims of our study were (i) to quantitatively investigate potential determining factors for children's nonparticipation and to what extent these contributed to the decision-making process and (ii) to qualitatively investigate children's subjective reasons for nonparticipation across different age spans.

\section{RESULTS}

\section{Quantitative Study}

Baseline characteristics. As the characteristics of the study participants were described elsewhere (5), we only give a brief overview here: of 209 children eligible for this study, 161 were enrolled, mean age 10.6 y (age range: 6-18 y).

Association of variables with participation. The distribution of the variables in contingency tables is demonstrated in Table 1. Sixty-two children (39\%) decided not to participate, 34 (21\%) did not make a decision on participation in the research project for which they were approached, and 64 children (40\%) decided to participate. Higher age, higher socioeconomic status, higher complexity and risk of the research procedure, Western ethnicity, and decision-making competence showed a positive association with the decision to participate $(P<0.01)$.

Strength of associations. The relative contribution of each characteristic to the probability of the decision to participate in research is listed in Table 2. High complexity and risk had the highest Wald statistic (13.6) and was most predictive for a decision to participate. Furthermore, greater disease and trial experience and a higher age contributed to a positive decision on trial participation (Wald statistic low experience 8.5, high experience 6.7; age 5.7). The Hosmer and Lemeshow goodness-of-fit $P$ value was: $P=0.24$. There were small differences between expected model probabilities and observed probabilities for the decision to participate (overall correct $>76 \%$ ).
Accuracy of associated variables. We further examined the predictive value of explanatory variables using area under the receiver operating characteristic curve (AUC) as a measure of accuracy: complexity and risk of the research procedure had an AUC of 0.61 (95\% confidence interval: 0.52-0.70); age 0.61 (95\% confidence interval: $0.51-0.70$ ); disease experience 0.58 (0.48-0.67). The AUC of all three explanatory variables combined in the model was 0.77 (95\% confidence interval: 0.690.85 ; Figure 1).

\section{Qualitative Study}

Thirty-five children were included in the qualitative study (mean age: 11.9 y, SD: 2.45 , age range: $8-16$ y). In this subsample, 25 children $(71 \%)$ decided to participate in the clinical research on offer.

Reasons for not participating. Children aged $9 \mathrm{y}$ and older presented 18 reasons for not participating in research. These reasons related to expected burdens for themselves directly deriving from research procedures, such as extra time investment, possible adverse effects of trial medication, or the inconvenience of sitting still. In seven cases, these reasons were decisive for children not to participate. Children aged 8 y did not express any reasons for not participating.

Several children all aged 9 y and older expressed 10 collateral burdens pertaining to themselves. No child explicitly mentioned a collateral burden for others. Many children mentioned that participating would impact on their time schedule, and children of all ages mentioned they did not want to miss school. Personal beliefs for not participating were expressed by children of $10 \mathrm{y}$ and older. Some children simply expressed they did not feel like participating.

Reasons for participating. On 40 occasions, children mentioned that participation would benefit others; some children expressed this more than once. To help advance knowledge for physicians was mentioned three times. Helping others was mentioned by children of all ages; in children aged $10 \mathrm{y}$ and older, helping others was frequently the decisive reason for participation.

In 11 cases, children, aged 8-13 y, mistook trial participation for individualized treatment. Children older than $13 \mathrm{y}$ did not express such a direct benefit. An example of a 11-y-old child asked to participate in research on treatment methods for functional abdominal pain clearly shows the child's misconception of the trial's purpose:

Interviewer: "Why would you rather take part?

Child: "Well, because the doctor told me there is an $80 \%$ chance that you succeed with the therapist, and... Yes, I really want my stomach ache to go away."

Only two children, aged 8 and 16 y, respectively, correctly interpreted the direct benefits they would receive from participating in the research. The following quote by the 16-y-old child participating in a study in gastroenterology illustrates this perfectly:

Interviewer: "Why would you rather take part?" 
Table 1. Distribution of variables among children who participate and children who do not

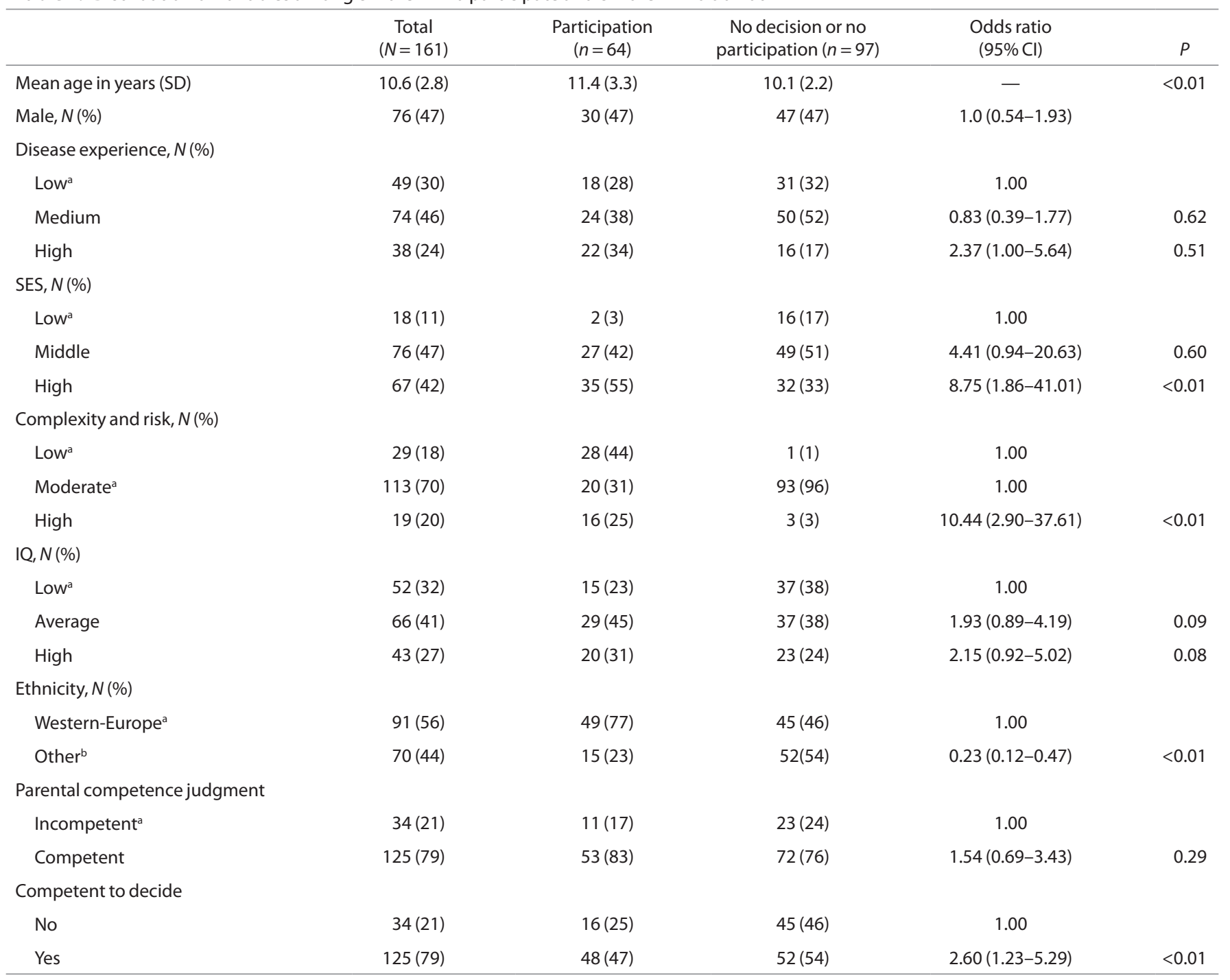

$\mathrm{Cl}$, confidence interval; IQ, intelligence quotient; SES, socioeconomic status.

a(Combined) reference category. ${ }^{~}$ Other: Middle East (30\%), Surinam/Antilles (13\%), and "other" $(1 \%)$.

Child: "Well, maybe they can find a solution for it that, yeah... mainly generally. And in the end maybe for me too."

Eight children of $10 \mathrm{y}$ and older mentioned collateral benefits for themselves, including the participation experience, which they considered fun, interesting, and instructive; however, younger children failed to mention these kind of motives altogether. Children of all ages expressed the belief that the research would not cause them any harm as a reason for them to participate. Personal beliefs concerning trial participation were mentioned by children of all ages (in 21 cases): common responses were, "I did it before," "it is your own choice," "I signed already, it is safe."

\section{DISCUSSION}

The quantitative study on potential determining factors for children's research participation showed that a lower age, less experience with disease and research, and less complex decisions with lower risk were all decisive factors not to participate in research. The percentage of nonparticipation was $60 \%$, of which $39 \%$ decided not to participate, while $21 \%$ remained indecisive. Although these results are solely based on the child's decision-in children under the age of $12 \mathrm{y}$, the parent's decision would be decisive-this percentage of nonparticipation is remarkably high. Research procedures in this study with more complexity and a higher level of risk were performed in pediatric oncology and one study in gastroenterology. The outcomes of prior studies are similar to our findings (6). Possible explanations for the higher participation rate in these cases point to the fact that families with healthcare experience may be more willing to allow their child to be enrolled in research. Furthermore, a conceivable motivation of seriously ill children to participate in research may be their higher dependence on the healthcare system and their search for hope. Moreover, children who had previously participated in studies, or families who were well informed about pediatric studies by active patient associations, were more willing to participate (6). Thus, 
Table 2. Association of variables with research participation

\begin{tabular}{|c|c|c|c|}
\hline & B & Wald & $\begin{array}{l}\text { Corrected odds } \\
\text { ratio }(95 \% \mathrm{Cl})\end{array}$ \\
\hline Age & 0.24 & 5.67 & $1.3(1.0-1.5)$ \\
\hline Gender & 0.20 & 0.22 & $1.2(0.5-2.8)$ \\
\hline \multicolumn{4}{|c|}{ Disease experience } \\
\hline Low $^{\mathrm{a}}$ & 0 & 8.52 & 0 \\
\hline Medium & 0.14 & 0.09 & $1.1(0.5-2.9)$ \\
\hline High & 1.43 & 6.66 & $4.2(1.4-12.3)$ \\
\hline \multicolumn{4}{|l|}{ SES } \\
\hline Low $^{\mathrm{a}}$ & 0 & 2.37 & 0 \\
\hline Middle & 1.00 & 1.07 & $2.7(0.4-17.8)$ \\
\hline High & 1.52 & 2.12 & $4.5(0.6-34.9)$ \\
\hline \multicolumn{4}{|c|}{ Complexity and risk } \\
\hline Low $^{\text {a }}$ & 0 & 0 & 0 \\
\hline Moderate $^{\mathrm{a}}$ & 0 & 0 & 0 \\
\hline High & 2.81 & 13.60 & $16.8(3.7-75.2)$ \\
\hline \multicolumn{4}{|l|}{ IQ } \\
\hline Low $^{\text {a }}$ & 0 & 0.45 & 0 \\
\hline Average & 0.25 & 0.21 & $1.3(0.5-3.6)$ \\
\hline High & 0.41 & 0.45 & $1.5(0.5-4.9)$ \\
\hline \multicolumn{4}{|l|}{ Ethnicity } \\
\hline Western $^{a}$ & 0 & - & 0 \\
\hline Other & -0.54 & 1.14 & $0.6(0.2-1.6)$ \\
\hline \multicolumn{4}{|c|}{ Parental competence judgment } \\
\hline Incompetent ${ }^{a}$ & 0 & - & 0 \\
\hline Competent & -0.16 & 0.06 & $0.9(0.2-3.1)$ \\
\hline \multicolumn{4}{|c|}{ Competent to decide } \\
\hline $\mathrm{No}^{\mathrm{a}}$ & 0 & 0 & 0 \\
\hline Yes & 0.28 & 0.23 & $1.3(0.4-4.1)$ \\
\hline
\end{tabular}

$\mathrm{Cl}$, confidence interval; IQ, intelligence quotient; SES, socioeconomic status. a(Combined) reference category.

familiarity may be an important factor in the decision on research participation.

Less willingness to participate among younger children may not be surprising. In previous studies, parents expressed that a restricted understanding of a study and its regulations was an important factor in deciding not to have their child participate in the study (6). Younger children may be equally discouraged if they do not fully understand the impact of the decision to participate in a study. Information provision directed at parents, and clear, age-appropriate information for children, may then help to improve the willingness to participate. Another factor may be that parents raising young children can feel overwhelmed, particularly when their child is sick. Minimizing the burden of research procedures in practical ways, for instance, by promoting online visits and telephone interviews instead of outpatient visits, might help relieve some of the pressure.

The other examined factors such as gender, socioeconomic status, intelligence, ethnicity, and parental judgment of a child's

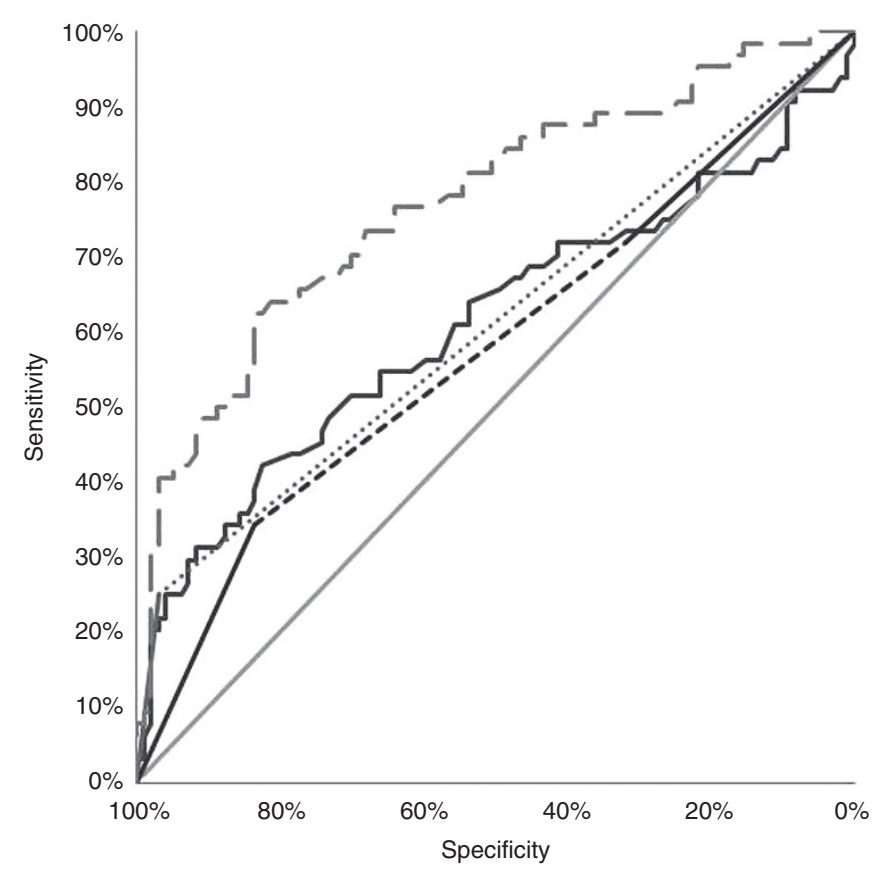

Figure 1. ROC curves of factors predictive for a decision to participate in research. Straight line, reference line; dashed line, experience; dotted line, complexity and risk; black irregular line, age; and gray dashed irregular line, combined. ROC, receiver operating characteristic.

competence to consent did not contribute significantly to promote or decline research participation.

The qualitative study on reasons that promote or discourage research participation expressed by children of different ages demonstrated that time constraints and direct burdens from the research procedures, such as waiting, sitting still, or extra tests, were the main reasons for not participating for children of aged $9 \mathrm{y}$ and older. Research procedures that were considered burdensome by children varied between individuals. In our sample, there were no trial procedures that required extra vena punctures or lumbar punctures-procedures involved electroencephalography, electrocardiography, magnetic resonance imaging, extra medication, diaries, and anorectal manometry. In general, children older than $9 \mathrm{y}$ were able to clearly convey their assessment on burdens and benefits.

Altruism was a main reason for children of $10 \mathrm{y}$ and older to participate in research. This corresponds to findings in the adult population (16). Another main reason was that children mistook research for individual treatment and expected health gain from participating in the research. This therapeutic misconception was prevalent in the population of children younger than $13 \mathrm{y}$, but not in late adolescents. In adults, therapeutic misconception emerged as a major theme (16); in previous studies in children, it was prevalent as well (13). Thus, in children, understanding of the purpose of clinical research relies heavily on the provision of information, which should be tailored to their comprehension level (17). Although children in our sample were well informed about the purpose of the research, including an explanation of the difference between therapeutic goals and research goals, younger children and early adolescents showed difficulties understanding. 
The results of both the qualitative and quantitative study show that the motives and characteristics of children who decide not to participate are the following: younger age, unfamiliar with healthcare and research, logistically challenged, not optimally informed, and not fully aware of the societal benefits. Strategies to improve recruitment rates could be targeted especially at pediatric patients and their families who fall into these categories. An example of an effective strategy to increase awareness of shared societal responsibility for trial recruitment can be found in adult studies (7) where public campaigns were recommended to convince professionals and the general public of the value of research for clinical practice. This included informing adult patients about the use of data to help future treatment and efforts to improve patient care (7). These strategies might also prove effective with pediatric patients and their parents.

Strategies for decreasing the logistic burden for children and families may be aimed at improving accessibility; the burden of time constraint should be addressed and minimized. Furthermore, as the majority of children older than $9 \mathrm{y}$ were able to convey their assessment on burdens and benefits in a clear way, it might be constructive to give children a voice in the assessment of what they experience as burdensome and valuable in research. An example is the Young Person's Advisory Board of the UK Medicines for Children Research Network (http:// www.crn.nihr.ac.uk/children/pcpie/young-persons-advisorygroup/), consisting of children, aged 8-18 y, who have experienced medical conditions. The board involves these children and families in research and raises research awareness and motivation among young people. Expanding similar initiatives may contribute to improving pediatric research recruitment rates.

\section{Limitations}

The population of participants was a heterogeneous group, which may limit the generalizability of the results to specific populations of children. Furthermore, it is a limitation that the parental view and its possible impact on the child is not included in this study. Another limitation may be caused by the decision to combine studies into low, middle, or high classifications of complexity and risk. Safety regulation warrants no more than minor increase over minimal risk for children in research. However, we anticipated the decision on research participation to be weightier in more seriously diseased children like those with cancer, thus we classified those in the high category, together with subjectively disagreeable research procedures, and the more complicated procedures. Unfortunately, levels of risk and complexity are not yet well defined or quantifiable $(18,19)$. The same limitation is valid for combining trial experience with duration of illness. Both familiarity with having a chronic disease as well as prior research participation are supposed to add to the child's experience, but such levels of experience are not well defined in literature either.

Finally, the role of the researcher fell outside the scope of this study. Researchers themselves, or the relationship between pediatric patients and researchers, might have specific characteristics that could increase or decrease the recruitment rate of studies.

\section{Conclusion}

Younger children, children with less disease experience, and children deciding on participation in less complex research with less risk were more prone to decline research participation. Time constraints and direct burdens from the research procedures were the main reasons for not participating expressed by children of $9 \mathrm{y}$ and older. Altruism was a subjective reason for research participation in children aged 10-18 y, and well-informed adolescents of $14 \mathrm{y}$ and older were not subject to therapeutic misconceptions.

Unfamiliarity, limited information provision for parents and young children, and logistic burdens are factors that negatively affect research participation; therefore, strategies should be aimed at these issues. Campaigns directed at convincing the professionals and general public of the value of research for clinical practice should include informing patients about the use of data and efforts to improve quality when entering the hospital. Logistic burdens should be minimized by coaching and guiding of children and parents and by improving accessibility. Involving children and families in advisory boards is a way to improve research awareness among eligible participants and to align research procedures with the participants' preferences.

Directives for future research include evaluation of the actual experience of research participation as well as research on how pediatric patients and their parents evaluate proportionality in decisions on research participation.

\section{METHODS}

\section{Participants}

The population for this study consisted of participants in a large study on competence to give informed consent in children, which is described comprehensively elsewhere (17). Between 1 January 2012 and 1 January 2014, this population of 161 pediatric patients, aged 6-18 y, eligible for real-life clinical research participation were prospectively recruited at inpatient and outpatient clinics in Amsterdam, Rotterdam, and The Hague (5). The clinical research projects consisted of 10 randomized controlled trials and three observational studies at departments of pediatric oncology, pediatric gastroenterology, internal medicine, ophthalmology, and pulmonary diseases. Only nonnative Dutch speakers were excluded from research participation.

For the quantitative study, all subjects were included; for the qualitative study, a sample consisting of every first enrolled participant of each elected age group ( $8-16 \mathrm{y})$ of each of the 10 research projects was selected, which would allow to aggregate sufficient data for analysis.

The study procedures were judged and approved by the institutional review boards of the participating institutions. Prior written informed consent was obtained from parents of participants and from participants aged $12 \mathrm{y}$ and older.

\section{Assessment Method}

Quantitative. Next to demographic data, we collected measures of potential determining factors: age; gender; experience, expressed in the number of trials previously participated in and duration of disease (low: no prior trial participation and duration of disease less than 1 mo; moderate: no prior trial participation and duration of disease more than, or equal to, 1 mo; high: prior trial experience); socioeconomic status, indicated by the level of education of the highest educated parent (low: no primary school, primary school, special primary school, special secondary school; middle: preparatory education, secondary vocational education, senior general secondary school, preparatory scientific education; high: college, university); complexity and 
risk of the research procedure (low: no risk, no blinding; moderate: little risk, placebo, and blinding; high: possible risk, complex or disagreeable research procedure, serious disease, placebo, and blinding); intelligence quotient, assessed by the Wechsler Nonverbal Scale of Ability, short version administered by trained certified professionals (low: under 90; average: 90-110; high: 110 or higher); and ethnicity (Western European, Middle East, Surinam/Antillean, or other). We used the MacArthur Competence Assessment Tool for Clinical Research (MacCAT-CR) modified for children $(5,17)$, and a semistructured interview format to assess children's decision-making competence concerning research participation. We also asked the parents to assess their own children's decision-making competence concerning research participation. Finally, we collected the child's own decision on participation in the research project on offer.

Qualitative. The MacCAT-CR understanding section disclosed information and provided questions on the children's comprehension of the purpose of the clinical research, the duration, the procedures, the research objectives, blinding in different treatment arms and the possibility of receiving placebo, and possible risks and benefits. The MacCAT-CR reasoning section contained questions on children's thoughts about research participation: what would you rather do, participate or not?; why would you rather participate, or not participate?; what do you think is good about participating, and what is not good about it?; why would you prefer participating (or not participating) above not participating (or participating)?

\section{Data Analysis}

Quantitative. We used multiple logistic regression analysis to examine to what extent the possible explanatory variables-age, gender, disease experience, socioeconomic status, complexity and risk of the study, intelligence, ethnicity, decision-making competence, and parental competence judgment-were related to the decision to participate, or not participate, in the research project. First, we examined the distribution of these variables in the sample in contingency tables. Ethnicity classifications were collapsed into Western European and other ethnicity. Then, all explanatory variables were entered simultaneously into the model to examine which variable contributed most to the probability for a positive decision on trial participation. Since adjusted odds ratio for the variables depend on their scale of measurement, we used the Wald statistic as a measure of strength of the association with trial participation.

Calibration of the model was examined using the procedure of Hosmer and Lemeshow (20) to test the differences between expected model probabilities and observed probabilities of the decision to participate in research. A Hosmer and Lemeshow test $P$ value $>0.05$ indicates no statistically significant differences between observed and expected frequencies regarding the decision to participate in research and thus the model fits acceptably. Accuracy of the model was examined by a receiver operating characteristic curve analysis. The AUC served as the validity coefficient; AUCs exceeding 0.70 are generally considered adequate.

Qualitative. The interviews were transcribed verbatim and checked against the recordings. We used the software package MAXQDA (VERBI GmbH, Berlin, Germany) to manage data. Two researchers (Lotte Gelens and I.M.H.) read and annotated five transcripts independently with no predetermined coding structure. We compared the transcripts to identify emergent themes and to identify initial codes. Further discussion identified predominant themes. The codes agreed upon were: reasons to participate; expected benefit; reasons not to participate; expected burdens; decisive reason; personal beliefs; probable choice; therapeutic misconception (conceptualizing research as treatment); and expected impact of participation. Each researcher then coded the remaining transcripts, while looking for consistencies to increase trustworthiness within and between transcripts. To illustrate the interviewees' responses, we extracted some common considerations mentioned by the participants, which were presented in the Results section.

\section{ACKNOWLEDGMENTS}

We thank Marjolaine Oosterbeek, junior researcher at Impact, Arq Foundation, Diemen, for her assistance with MAXQDA. Many thanks are owed to
Lotte Gelens, research assistant at Academic Medical Center, Amsterdam, for assisting in data collection.

\section{STATEMENT OF FINANCIAL SUPPORT}

All phases of this study were supported by a grant from ZonMW, The Netherlands Organization for Health Research and Development, number 11-3105-006.

Disclosure: All authors have no financial relationships relevant to this article to disclose.

\section{REFERENCES}

1. Cuzzolin L, Atzei A, Fanos V. Off-label and unlicensed prescribing for newborns and children in different settings: a review of the literature and a consideration about drug safety. Expert Opin Drug Saf 2006;5: 703-18.

2. Wendler D, Belsky L, Thompson KM, Emanuel EJ. Quantifying the federal minimal risk standard: implications for pediatric research without a prospect of direct benefit. JAMA 2005;294:826-32.

3. United States Department of Health and Human Services. Code of Federal Regulations. 45 CFR 46:408. Federal Policy for the Protection of Human Subjects (Subpart D). Washington, DC: United States Department of Health and Human Services, 2009.

4. Wendler D, Shah S. Should children decide whether they are enrolled in nonbeneficial research? Am J Bioeth 2003;3:1-7.

5. Hein IM, Troost PW, Lindeboom R, et al. Accuracy of the MacArthur competence assessment tool for clinical research (MacCAT-CR) for measuring children's competence to consent to clinical research. JAMA Pediatr 2014;168:1147-53.

6. Vanhelst J, Hardy L, Bert D, et al. Effect of child health status on parents' allowing children to participate in pediatric research. BMC Med Ethics 2013;14:7.

7. Oude Rengerink K. Embedding Trials in Evidence-Based Clinical Practice. Amsterdam: UvA-DARE, 2014.

8. Cox SM, McDonald M. Ethics is for human subjects too: participant perspectives on responsibility in health research. Soc Sci Med 2013;98:224-31.

9. Barakat LP, Patterson CA, Mondestin V, et al. Initial development of a questionnaire evaluating perceived benefits and barriers to pediatric clinical trials participation. Contemp Clin Trials 2013;34:218-26.

10. Wulf F, Krasuska M, Bullinger G. Determinants of decision-making and patient participation in peadiatrc clinical trials: a literature review. Open J Pediatr 2012;2:2-17.

11. Brody JL, Turner CW, Annett RD, Scherer DG, Dalen J. Predicting adolescent asthma research participation decisions from a structural equations model of protocol factors. J Adolesc Health 2012;51:252-8.

12. Miller VA, Baker JN, Leek AC, et al. Adolescent perspectives on phase I cancer research. Pediatr Blood Cancer 2013;60:873-8.

13. Unguru Y, Sill AM, Kamani N. The experiences of children enrolled in pediatric oncology research: implications for assent. Pediatrics 2010;125:e876-83.

14. Wendler D, Abdoler E, Wiener L, Grady C. Views of adolescents and parents on pediatric research without the potential for clinical benefit. Pediatrics 2012;130:692-9.

15. Reed JL, Thistlethwaite JM, Huppert JS. STI research: recruiting an unbiased sample. J Adolesc Health 2007;41:14-8.

16. Townsend A, Cox SM. Accessing health services through the back door: a qualitative interview study investigating reasons why people participate in health research in Canada. BMC Med Ethics 2013;14:40.

17. Hein IM, Troost PW, Lindeboom R, de Vries MC, Zwaan CM, Lindauer RJ. Assessing children's competence to consent in research by a standardized tool: a validity study. BMC Pediatr 2012;12:156.

18. Alderson P. Competent children? Minors' consent to health care treatment and research. Soc Sci Med 2007;65:2272-83.

19. Scherer DG, Annett RD, Brody JL. Ethical issues in adolescent and parent informed consent for pediatric asthma research participation. J Asthma 2007;44:489-96.

20. Hosmer DW, Lemeshow S. Applied Logistic Regression. New York: Wiley, 2014. 